\title{
Progressive lymphocytic-plasmocytic enteritis in a horse
}

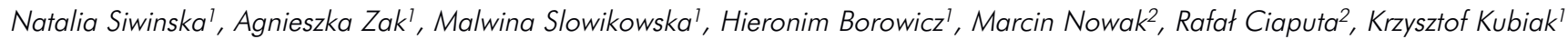 \\ and Artur Niedzwiedz ${ }^{7}$ \\ 1 Department of Internal Medicine and Clinic of Diseases of Horses, Dogs and Cats, Faculty of Veterinary Medicine, Wroclaw University of Environmental and \\ Life Sciences, Poland \\ 2 Division of Pathomorphology and Veterinary Forensics, Department of Pathology, Faculty of Veterinary Medicine, Wroclaw University of Environmental and Life \\ Sciences, Poland.
}

\begin{abstract}
Summary: A 14-year old Polish half bred mare, with a three months history of weight loss and recurrent colic was referred to the clinic. A series of diagnostic tests were performed, including glucose absorption test, abdominal ultrasound examination, abdominocentesis, endoscopy with duodenum biopsy and a rectal biopsy. Complete examination revealed a moderate inflammatory infiltrate consisting of lymphocytes and plasma cells in the lamina propria in large intestine and peritonitis. The horse was treated with dexamethasone, anthelmintics and a proper diet. The mare initially responded well to the medication course. Clinical signs of colic reappeared during treatment, and there was no response to analgesic and antispasmodic drugs. The owner elected euthanasia. Histopathology at necropsy confirmed progression of lymphocytic-plasmacytic enteritis in all parts of intestine. In the presented horse, there was an exacerbation of the disease after arrival at the Clinic, which may be associated with the development of the disease or its earlier asymptomatic advance. Due to the diagnostic difficulties associated with this condition, it may be undiagnosed in a large number of horses suffering from recurrent colic and weight loss. The exact cause and predisposition to develop IBD is not diagnosed in all horses. Further studies are needed to enable early diagnosis and the introduction of an effective treatment.
\end{abstract}

Keywords: equine, lymphocytic-plasmacytic enteritis, weight loss, inflammatory bowel disease, colic

Citation: Siwinska N., Zak A., Slowikowska M., Borowicz H., Nowak M, Ciaputa R., Kubiak K., Niedzwiedz A.(2017) Progressive lymphocytic-plasmocytic enteritis in a horse. Pferdeheilkunde 33, 252-256; DOI 10.21836/PEM20170505

Correspondence: Natalia Siwinska, DVM, PhD, Department of Internal Diseases, The Faculty of Veterinary Medicine, Wroclaw University of Environmental and Life Sciences, PI. Grunwaldzki 47, 50-366 Wroclaw, Lower Silesia, Poland; e-mail address: natalia.siwinska @upwr.edu.pl.

\section{Introduction}

Lymphocytic-plasmacytic enteritis (LPE) is one of the diseases composing the inflammatory bowel disease (IBD) and leads to various degrees of maldigestion and malabsorption. This condition is characterized by large lymphocyte and/or plasma cell infiltration in the intestinal lamina propria. LPE is one of the most common types of bowel inflammation in dogs and cats (Schumacher et al. (2000)). However, there have been only a few reports of LPE in horses (Clark et al. (1988), Kaikkonen et al. (2014), Kemper et al. (2000), MacAllister et al. (1990), Scott et al. (1999)). In this case report, the authors describe a very well documented case of lymphocytic-plasmacytic enteritis, supplemented by the description of the presentation of the disease, which, to date, has not been described in horses. To the authors' best knowledge, none of previous presented cases described so rapid progression of disease. Compared to the other cases available in the literature, the large intestine of the horse was affected and IBD was accompanied by peritonitis. These two clinical symptoms are not usual in LPE in horses. The article highlights the diagnostic and treatment challenges in equine inflammatory bowel disease, based on single clinical case.

\section{Case report}

A 14 year old Polish half bred mare was brought to the Department of Internal Medicine and Clinic of Diseases of Horses, Dogs and Cats due to progressive weight loss and recurrent mild to moderate colic lasting three months. The
Henneke body condition score was 3/9 (Henneke et al. 1983). The animal was depressed and had an inconsistent appetite. The vital signs of the horse were within normal limits. The blood biochemistry revealed a decreased concentration of total protein, reaching $54 \mathrm{~g} / \mathrm{l}$. The remaining blood parameters were normal. The examination of the oral cavity and transrectal palpation were unremarkable. The positioning and consistency of the abdominal organs was normal, and there was no pain on palpation. There were no parasite eggs in the sample examined using the fecal flotation test. There was no sand in the feces. Gastroscopy of the upper digestive tract excluded the presence of inflammation or ulceration in the stomach or duodenum. The biopsy specimens taken from the pylorus for a histological examination did not reveal any pathological changes. An oral carbohydrate absorption test was also carried out using $1 \mathrm{~g} / \mathrm{kg}$ of body mass glucose in a $20 \%$ solution. The glucose was administered to the horse after 12 hours of fasting through a nasogastric tube. The glucose concentration was monitored with a blood glucose meter used in humans, prior to glucose administration and every 30 minutes for six hours following glucose administration. The glucose concentration increased by $115 \%$ two hours later and returned to baseline levels within six hours. The results of the oral glucose absorption test were normal. A transabdominal ultrasound showed a significant increase in the level of peritoneal fluid, predominantly in the lower abdomen (Fig. 1A) and around parenchymal organs, particularly the spleen (Fig. 1B). The ultrasound images of the small and large intestines were normal with no evidence of wall thickening. An abdominocentesis was carried out in the lower abdomen and revealed pale straw-colored turbid peritoneal 
fluid. The specific gravity of the fluid was $1.008 \mathrm{~g} / \mathrm{ml}$, the total protein content was $6.3 \mathrm{~g} / \mathrm{l}$, and there were $52 \%$ neutrophils of total cell count, most of which were degenerated. The fluid indicated peritonitis. A bacterial infection was ruled out based on a microbiological examination. A rectal biopsy was carried out, and a rectal sample was collected. An examination of the sample revealed a moderate inflammatory infiltrate consisting of lymphocytes and plasma cells in the lamina propria. The horse was treated for inflammatory bowel disease with corticosteroids, anthelmintics and diet. Dexamethasone was administered at $0.1 \mathrm{mg} / \mathrm{kg}$, which was to be reduced later to a maintenance dose. The mare was dewormed using fenbendazole at $10 \mathrm{mg} / \mathrm{kg}$, administered on five consecutive days, then single dose of ivermectin with praziquantel. The horse received commercial St. Hippolyt Brandon XS fibrousprotein forage, intended to be used in skinny horses, horses

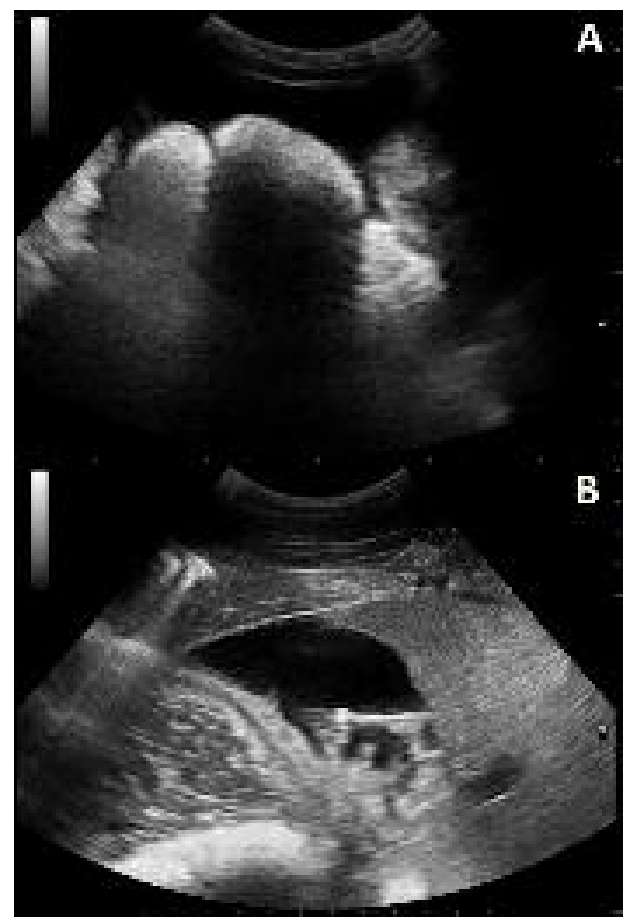

Fig.1 Ultrasonographic images of the abdomen from the mare. Image from the ventral midline window of abdomen document increase in the level of peritoneal fluid and normal intestinal wall thickness (A). Image from the left side of abdomen in gastric window evidence significant increase of the peritoneal fluid with fibrin between stomach and spleen (B).

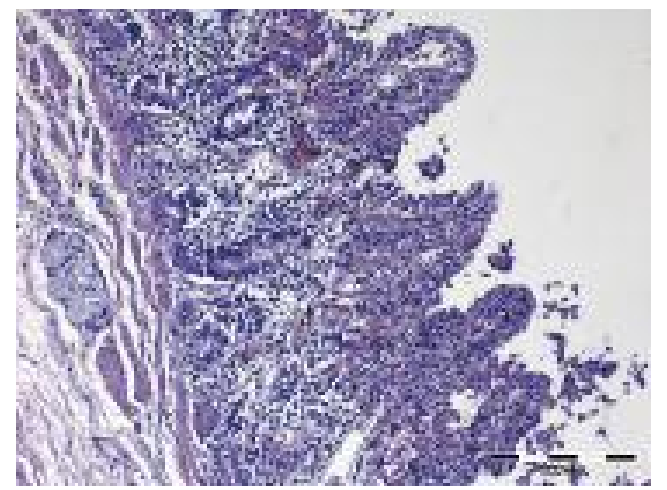

Fig.2 Representative histologic images of the mares' small intestinal wall documenting excessive inflammatory infiltrate in the lamina propria comprising lymphocytes and plasma cells. Visible reduction of intestinal villi. during convalescence and those with problems digesting and absorbing feed. The feed was dosed at $0.75 \mathrm{~kg}$ of feed per $100 \mathrm{~kg}$ of body mass divided into six small meals per day. The horse received high-quality hay as roughage, which was provided several times per day.

In the course of the treatment, the mare developed clinical signs of colic that intensified and did not respond to pain or spasmolytic treatment. Large colon torsion in the pelvic region was diagnosed in the rectal examination. The owner chose to euthanize the horse and consented to a post-mortem examination, which revealed a $360^{\circ}$ torsion of the left dorsal and ventral colon and diffuse macroscopic lesions in the wall of the small intestine. The intestinal wall had focal, diffuse changes in color. There were no palpable changes in the intestinal wall such as oedema. The innermost surface of the mucous membrane had no macroscopic lesions. The histopathological examination of the small intestine revealed an extensive lymphocytic infiltrate in the lamina propria, which confirmed the presence of lymphocytic enteritis (Fig.2).

\section{Discussion}

IBD should be included in the differentia diagnosis in horses with progressive weight loss and recurring colic. LPE is uncommon condition in horses. The authors did not suspect LPE to be a problem of clinical relevance, immediately after admitting the horse. A number of diagnosic tests were needed to rule out more common diseases. The clinical signs of disease are non-specific and include unexplained weight loss or no increase in body weight, recurrent colic symptoms, apathy or lethargy. The animal may have a normal or decreased appetite depending on the severity of the disease (Kalck 2009). Some patients may also suffer from chronic diarrhea (Kaikkonen et al. 2014), Schumacher et al. 2000). According to Durham and Rendle (2010) recurrent colic is the dominating symptom in all types of enteritis. Oedema and thickening of the intestinal wall may lead to a mechanical obstruction (Scott et al. 1999). Disorders of motility associated with chronic inflammation may cause displacement and torsion of the intestine (Samsel and Witkowski 2012). The most constant clinical sign in horses with LPE described in the literature is weight loss (Kaikkonen et al. (2014), Kemper et al. (2000), MacAllister et al. (1990). Diarrhoea is present only in $50 \%$ of horses with LPE (Kemper et al. 2000). Thickening of the intestinal wall, which may exceed $>4 \mathrm{~mm}$ in the ultrasound examination, is present in $50 \%$ of the IBD cases, but genuinely was seen only in 2 described cases of LPE (Durham and Rendle (2010), Kemper et al. (2000). The usefulness of the ultrasonographic examination is limited by the inability to visualise the affected intestine, most likely because of the segmental nature of the lesion (Kemper et al. 2000). In the present case, the only visible clinical signs of the disease were weight loss and recurrent colic, with no changes in the consistency of faeces or ultrasonographic thickening of the intestinal wall. Increase in the amount of abdominal fluid is rarely seen in the course of LPE (Kemper et al. 2000). However, it was present in the mare, indicating chronic peritonitis. Other likely causes of peritonitis were excluded.

The disease is quite difficult to diagnose because there are no changes in the blood and peritoneal fluid. Hypoproteinemia 
and disorders of carbohydrate digestion are not a constant symptom and may occur in the course of other pathological conditions (Samsel and Witkowski 2012). The oral carbohydrate absorption test is an easy indirect test assessing the functioning of the small intestine. In literature, the test was abnormal in $75 \%$ cases (Kemper et al. 2000). However, as in our case, it may give false results since it evaluates the absorptive function of the small intestine, not the function of the large intestine.

In horses and small animals the disease usually affects the small intestine. However, in the described horse, it also involved the large intestine, which signifies the progression of the disease (Couto et al. 1989, Dennis et al. 1992, Jacobs et al. 1990, Kemper et al. 2000). A rectal biopsy may not be a valuable diagnostic tool in the case of LPE (Kemper et al. 2000, MacAllister et al. 1990, Schumacher et al. 2000). In the reported case, the clinical changes were more pronounced in the large intestine than the small intestine and also involved the rectum. The number of cells within the intestinal wall varies depending on the area and shows inter-individual variation. Plasma cells and lymphocytes may be present in the rectum of horses in various diseases, not associated with LPE and their presence in the biopsy specimen does not fully confirm the disease (Kalck 2009). In a retrospective study carried out on 14 horses with lymphocytic/plasmacytic enteritis, the histopathological examination of specimens obtained endoscopically from the duodenum did not reveal inflammatory lesions (Kemper et al. 2000). This diagnostic tool often gives false negative results. The disease process may not include this part of digestive tract and most of the specimens thus obtained are usually superficial (Couto et al. 1989). This may explain the negative result of the histological examination of the pylorus biopsy obtained from the mare. The only reliable test confirming IBD ante-mortem is an analysis of a full-thickness bowel biopsy specimen, but is also invasive and expensive (Durham and Rendle 2010, Kemper et al. 2000). The specimen may be collected during a laparotomy or laparoscopy. In literature only in one of the horse with LPE, biopsy specimens of the small intestine were considered microscopically normal, however necropsy revealed LPE in this part of digestive tract (Kemper et al. 2000). In most cases, the diagnosis is confirmed post mortem.

The cause of an inflammatory infiltrate is unknown, and most likely results from an abnormal immune response to viral, bacterial, parasitic and dietary antigens (Dennis et al. 1992, Durham and Rendle 2010, Jacobs et al. 1990, Marks 1998). The digestive system is considered the largest component of the immune system (Chahine and Bahna 2010). A normal immune response is directed against microorganisms crossing the intestinal barrier. This active, selective barrier that does not react to antigens present in the intestinal lumen is known as "food tolerance". Food intolerance and an imbalanced intestinal flora result in continuous inflammation (Thibualt et al. 2010). Studies conducted in humans suffering from Crohn's disease showed differences between the microbiota of the sick and healthy persons (Dunn et al. 2016, Kaakoush et al. 2015, Qiao et al. 2016). Due to the fact that the cause of IBD in horses has not been confirmed, causative treatment of the disease is not possible. No predisposing factors for the development of LPE, such as age, gender, breed or diet were identified (Kemper et al. 2000).
Corticosteroids at a reduced dose, an antiparasitic therapy and a diet constitute the therapy of choice in IBD. During the initial stages of the disease dexamethasone is administered at $0.05-0.1 \mathrm{mg} / \mathrm{kg}$ intramuscularly or intravenously every 24 hours and then is tapered to the lowest maintenance dose, usually $0.02 \mathrm{mg} / \mathrm{kg}$ administered orally (Kaikkonen et al. 2014). An antiparasitic therapy is recommended regardless of the results of the parasitic examination (Kaikkonen et al. 2014, Lyons et al. 2000). Fenbendazole should be administered at $10 \mathrm{mg} / \mathrm{kg}$ for five successive days together with ivermectin and praziquantel (Kaikkonen et al. 2014). Fenbendazole administered at the above mentioned doses also acts against encysted Cyathostominae (Lyons et al. 2000). An easily digestible diet is recommended in all gastrointestinal diseases and should be administered frequently, in small portions. This improves digestion and the absorption of nutrients (Kalck 2009). The response of LPE to dietary modifications has not been studied in horses (Kemper et al. 2000). In human research, a large influence of dietary factors on the development of the disease has been noted (Qiao et al. 2016). IBD treatment is usually long-term (lasts four to eight weeks) or may be even life-long. The duration of the treatment and the corticosteroid dose is determined individually for each patient. An individual type of treatment program was applied to the horse treated in our clinic. The mare initially responded well to the treatment. She was alert and her appetite returned. However, she developed symptoms of colic. The colic volvulus may have been associated with the disease progression as well as other factors (such as dietary changes). The progression of the LPE was confirmed in a subsequent histopathological examination of specimens of the small and large intestine, where the inflammatory infiltrate was more extensive compared to the previous examination. The prognosis for inflammatory enteritis depends on the severity of the disease and the localization of the infiltration. The prognosis in the case of LPE is guarded, even if the treatment is introduced shortly after diagnosis. The advancement of the disease in the mare could have indicated a long lasting asymptomatic process. In small animals the transformation of LPE into intestinal lymphoma has been noted (Kemper et al. 2000, Jacobs et al. 1990). LPE is suspected to be an early form of lymphoma, but differentiating between those two conditions can be difficult (Couto et al. 1989, Dennis et al. 1992, Kalck 2009).

Studies assessing long-term survival in horses with IBD treated with corticosteroids show that horses with granulomatous enteritis responded to the treatment well, while the disease progressed despite treatment in horses with LPE (Kaikkonen et al. 2014). Similar results were obtained in the USA in 17 cases of horses with LPE, which were euthanized due to no response to treatment and a deteriorating physical condition (Kaikkonen et al. 2014, Kemper et al. 2000, MacAllister et al. 1990). Our findings and literature reports indicate that systemic corticosteroid therapy does not have a satisfactory therapeutic effect in the case of LPE in horses. This may be due to the asymptomatic duration of the process and, a late diagnosis. We also noted a significant progression of the disease prior to initiating treatment. An earlier displacement of the large colon prior to a volvulus cannot be ruled out. However, this seems unlikely as the clinical, rectal and ultrasound examination did not reveal the presence of colonic displacement or volvulus as a primary cause of the disease. 
Following admittance to the clinic, the horse did not manifest signs of colic pain. Long-term volvulus would also cause intestinal wall lesions (such as ischemia), which were not identified in the post-mortem examination.

Due to the differences in the survival of horses with LPE and other types of IBD, it may be suspected that LPE has a different pathomechanism than in small animals. Based on the small number of horses with LPE reported in literature and clinical practice, it appears that this disease is less common in horses than in small animals. Due to the diagnostic difficulties associated with LPE, it may be undiagnosed in a large population of horses. In new research in humans with IBD, transplantations of fecal microbiota restore the balance of the intestinal flora and optimize current therapies (Liang et al. 2014). Disruption of the intestinal flora may have diagnostic significance in the early stages of the disease, but there are no such reports in horses. Further studies on the etiology and mechanisms of LPE in horses are needed to enable early and precise diagnosis and the introduction of an effective treatment.

\section{References}

Chahine B., Bahna S. (2010) The role of the gut mucosal immunity in the development of tolerance verus development of allegry to food. Curr. Opin. Allergy Clin. Immunol. 10, 394-399

Clark E. S., Morris D. D., Allen D., Tyler D. E. (1988) Lymphocytic enteritis in a filly. J. Am. Vet. Med. Assoc. 10, 1281-1283

Couto C. G., Rutgers H. C., Sherding R. G., Rojko J. (1989) Gastrointestinal lymphoma in 20 dogs. A retrospective study. J. Vet. Int. Med. 3, 73-78

Dennis J. S., Kruger J. M., Mullaney T. P. (1992) Lymphocytic/plasmacytic gastroenteritis in cats: 14 cases (1985-1990). J. Am. Vet. Med. Assoc. 11, 1712-1718

Dunn K. A., Moore-Connors J., Maclntyre B., Stadnyk A., Thomas N. A., Noble A., Mahdi G., Rashid M., Otley A. R., Bielawski J. P., Van Limbergen J. (2016) The gut microbiome of pediatric Crohn's disease patients differs from healthy controls in genes that can influence the balance between a healthy and dysregulated immune response. Inflamm. Bowel Dis. 22, 2607-2618

Durham A. E., Rendle D. (2010) Inflammatory bowel disease as a cause of colic: diagnosis and treatment. Proceeding of the 49th British Equine Veterinary Association Congress - Birmingham, United Kingdom
Henneke D. R., Potter G. D., Kreider J. L., Yeates B. F. (1983) Relationship between condition score, physical measurements and body fat percentage in mare. Equine Vet. J. 15, 371-372

Jacobs G., Collins-Kelly L., Lappin M., Tyler D. (1990) Lymphocyticplasmacytic enteritis in 24 dogs. J. Vet. Int. Med. 4, 45-53

Kaakoush N. O., Day A. S., Leach S. T., Lemberh D. A., Nielsen S., Mitchell H. M. (2015) Effect of exclusive enteral nutrition on the microbiota of children with newly diagnosed Crohn's disease. Clin. Translation. Gastroenterol. 6, e71, DOI 10.1038/ctg.2014. 21

Kaikkonen R., Niinist K., Sykes B., Anttila M., Sankari S., Raekallio M. (2014) Diagnostic evaluation and short-term outcome as indicators of long-term prognosis in horses with findings suggestive of inflammatory bowel disease treated with corticosteroids and anthelmintics. Acta Vet. Scand. 56, 35

Kalck K. A. (2009) Inflammatory Bowel Disease in Horses. Veterinary Clinics of North America: Equine Pract. 25, 303-15

Kemper D. L., Perkins G. A., Schumacher J., Edwards J. F., Valentinen B. A., Divers T. J., Cohen N. D. (2000) Equine lymphocytic-plasmocytic enterocolitis: a retrospective study of 14 cases. Equine Vet. J.. Suppl. 32, 108-112

Liang J., Mei Sha S., Chun Wu K. (2014) Role of the intestinal microbiota and fecal transplantation in inflammatory bowel diseases. Digest. Dis. 15, 641-646

Lyons E. T., Drudge J. H., Tolliver S. C. (2000) Larval cyathostomiasis. Vet. Clin. North Am. Equine Pract. 16, 501-513

MacAllister C. G., Mosier D., Qualls C. W., Cowell R. L. (1990) Lymphocytic-plasmacytic enteritis in two horses. J. Am. Ve. Med.Assoc. 12, 1995-1998

Marks S. L. (1998) Management of canine inflammatory bowel disease. Comp. Cont. Educ. Pract. Vet. 3, 317-331

Qiao Y.Q., Cai C. W., Ran Z. H. (2016) Therapeutic modulation of the gut microbiota in IBD - more question to be answered. Digestive Dis. 17, 800-810

Samsel J., Witkowski M. (2012) Analiza przypadków eozynofilowego zapalenia jelita czczego i okr nicy u koni (Analysis of cases eosinophilic enterocolitis in horse). Zycie Weterynaryjne, Poland 87(8), 680-684

Schumacher J., Edwards J. E., Cohen N. D. (2000) Chronic Idiopathic Inflammatory Bowel Diseases of the Horse. J. Vet. Int. Med. 14, 258-265

Scott E. A., Heidel J. R., Snyder S. P., Ramirez S., Whitler W. A. (1999) Inflammatory bowel disease in horses: 11 cases (1988-1998). J. Am. Vet. Med. Assoc. 10, 1527-1530

Thibualt R., Blachier F., Darcy-Vrillon B., de Coppet P., Bourreille A., Segain J. P. (2010) Butyrate utilization by the colonic mucosa in inflammatory bowel disease: a transport deficiency. Inflamm. Bowel Dis. 16, 684-695 